\title{
Efficient control of mechatronic systems in dynamic motion tasks
}

\author{
Desislava Despotova, Petko Kiriazov*, and Stefan Karastanev \\ Institute of Mechanics, Bulgarian Academy of Sciences, Acad. G. Bonchev Str., bl.4, BG-1113 Sofia, \\ Bulgaria
}

\begin{abstract}
Robots and powered exoskeletons have often complex and nonlinear dynamics due to friction, elasticity, and changing load. The proposed study addresses various-type robots that have to perform dynamic point-topoint motion tasks (PTPMT). The performance demands are for faster motion, higher positioning accuracy, and lower energy consumption. With given motion task, it is of primary importance to study the structure and controllability of the corresponding controlled system. The following natural decentralized controllability condition is assumed: the signs of any control input and the corresponding output (the acceleration) are the same, at least when the control input is at its maximum absolute value. Then we find explicit necessary and sufficient conditions on the control transfer matrix that can guarantee robust controllability in the face of arbitrary, but bounded disturbances. Further on, we propose a generic optimisation approach for control learning synthesis of various type robotic systems in PTPMT. Our procedure for iterative learning control (LC) has the following main steps: (1) choose a set of appropriate test control functions; (2) define the most relevant input-output pairs; and (3) solve shooting equations and perform control parameter optimisation. We will give several examples to explain our controllability and optimisation concepts.
\end{abstract}

\section{Introduction}

Controlled mechanical systems (CMS) are various-type robotic systems [1,2], vehicles/platforms with active suspension $[3,4]$, and other engineering structures with active vibration/shape control. CMS have to be considered as functionally directed compositions of mutually influencing subsystems: control, actuator, structural, and sensor subsystems. Such systems have highly complex dynamics and advanced conceptual frameworks are needed that consider at the same time the problems of full dynamic modelling, optimal system design, accurate parameter identification, and optimal robust control. There are various design tasks for controlled mechanical systems (CMS), where continuously increasing demands for higher speed, better motion accuracy, and reduced energy consumption are to be satisfied. In order to achieve such complicated performance optimization, it is very important to study their controllability and find efficient solutions for the control-related, design optimization problems. Our intention is to present novel concepts and criteria for design optimization of CMS having, as usual, decentralized controllers at the lowest (joint) level. The following natural decentralized controllability 
condition is assumed: the signs of any control input and the corresponding output (the acceleration) are the same, at least when the control input is at its maximum absolute value.

In optimal feedback control problems, one has in principle to optimize between error and energy for finding the most appropriate control gains. In the well-known control methods using linear-quadratic or H-infinity optimization functionals [4], the weight matrices are critical towards the required system performance. Choosing their coefficients only by trial-and-error, however, may not guarantee achievement of the required motion accuracy within the existing control power limits. Moreover, optimality and robustness cannot be guaranteed if a control design method does not take into account a realistic estimate for modelling errors and system disturbances, [5].

In this paper we present a generic design and control optimization approach, which has the potential to overcome any of the above problems in an efficient way. CMS of varioustype can be considered with the premise that they can be equipped with a sufficiently large number of actuators. Our concept is that system performance that is optimal and robust to arbitrary-in-place external disturbances is hard to provide if the actuators' number is smaller than the number of the controlled performance variables.

The first step is to find a dynamic model relating the control inputs and the controlled outputs which is suitable for both purposes: accurate identification and robust control design. To do that, we can apply the so-called multibody system (MBS) approach: the mechanical structure of CMS can be approximated by a composition of rigid bodies connected by joints, actuators, springs, and dampers [6]. Then we find explicit necessary and sufficient conditions on the control transfer matrix that can guarantee robust controllability in the face of arbitrary, but bounded disturbances. Thus the design optimization process has to involve, besides the basic strength/load capacity criterion, additional design relations for optimal trade-off between the bounds of disturbances and the control force limits [7,8]. Further on, we propose a generic optimisation approach for control learning synthesis of various type robotic systems in dynamic point-to-point motion tasks (PTPMT) Our procedure for iterative learning control (LC) has the following main steps: (1) choose a set of appropriate test control functions; (2) define the most relevant input-output pairs; and (3) solve shooting equations and perform control parameter optimisation. We will give several examples to explain our controllability and optimisation concepts.

\section{Controllability of multi-joint dynamics}

First, it is very important to properly define the controls, the performance variables to be controlled, and the structure of the input-output relations, [9]. We have to find nominal dynamic models that are appropriate for both purposes, accurate parameter identification [10] and robust control design, [11]. As said above, any mechanical system can be approximated, in general, by a composition of rigid bodies connected by joints, springs, dampers, and driving forces. Applying the Lagrange formalism, the dynamic performance of such a MBS can be described by

$$
\ddot{\mathbf{q}}=\mathbf{M}^{-1}(\mathbf{q})(\mathbf{B u}-\mathbf{C}(\mathbf{q}, \dot{\mathbf{q}})+\mathbf{g}(\mathbf{q})),
$$

where $\mathbf{q}$ is the vector of performance variables/controlled outputs, $\mathbf{M}$ is the inertia matrix, $\mathbf{C}$ is the vector of velocity forces, $\mathbf{g}$ stands for friction and gravitation forces, matrix $\mathbf{B}$ represents the actuator locations/gains, and $\mathbf{u}$ is the vector of control inputs (forces, torques). 
Thus for MBS with elastic elements, dynamic model structures can be described by a relatively small number of parameters. All coefficients in (1) can be identified in a systematic way as proposed in [10].

In principle, using both feedforward and feedback control functions is needed for optimal performance to be achieved. With a feedforward control, we can compensate, to some extent, for the gravitation, stiffness and other forces. As usual, we have decentralized controllers at the lowest (joint) level and the control magnitudes $\left|\mathbf{u}_{i}\right|$ should fulfil the following natural condition for independent joint controllability:

$$
\operatorname{sign} \ddot{\mathbf{q}}_{i}=\operatorname{sign} \mathbf{u}_{i}, \quad \text { if } \quad\left|\mathbf{u}_{i}\right|=\overline{\mathbf{u}}_{i}, i=1, \ldots, n .
$$

For the purpose of feedback control design, the following simple, but sufficiently general, model for the error dynamics can be used:

$$
\ddot{\mathbf{e}}=\mathbf{A}(\mathbf{q}) \mathbf{u}+\mathbf{d},
$$

where: $\ddot{\mathbf{e}}=\mathbf{q}-\mathbf{q}^{\text {ref }}$; vector $\mathbf{d}$ stands for the model imprecision and the uncompensated terms as well as for the measurement and environment noises; $\mathbf{A}=\mathbf{M}^{-1}(\mathbf{q}) \mathbf{B}$ is the control transfer matrix which will play a central role in the next control design considerations.

A necessary and sufficient condition for the dynamic system (1) to be robustly controlled by a decentralized controller is that matrix $\mathbf{A}$ be generalized diagonally dominant (GDD), [11]. It can be easily verified that any mechanical system with two degrees of freedom has control transfer matrix which is GDD.

$$
c_{i} \mathbf{A}_{i j}>\sum_{j \neq i} c_{j}\left|\mathbf{A}_{i j}\right| \text {, for some } c_{i}>0, i=1, \ldots, n .
$$

With A being GDD, the non-negative matrix theory [12] states that there always exists a positive vector $\overline{\mathbf{u}}$ of control magnitudes that solve the following system of equations

$$
\mathbf{A}_{i i} \overline{\mathbf{u}}_{i}-\sum_{j \neq i}\left|\mathbf{A}_{i j}\right| \overline{\mathbf{u}}_{j}=\overline{\mathbf{d}}_{i},
$$

where $\overline{\mathbf{d}}_{i}$ are some upper bounds for the disturbances.

Eqs. (5) present optimal trade-off relations between the bounds of model uncertainties and the control force limits. The greater the determinant $\Delta$ of this system of linear equations, the less control forces required to overcome the disturbances. Therefore, the index $\Delta$ can be used to analyze, measure, and optimize the independent joint controllability.

\section{Examples on how controllability can be improved}

\subsection{Two-link manipulator/exoskeleton}

Consider a two-degree-of-freedom manipulator and assume, for simplicity, that the masses $m_{1}$ and $m_{2}$ of the links are concentrated at their ends, Fig. 1. 


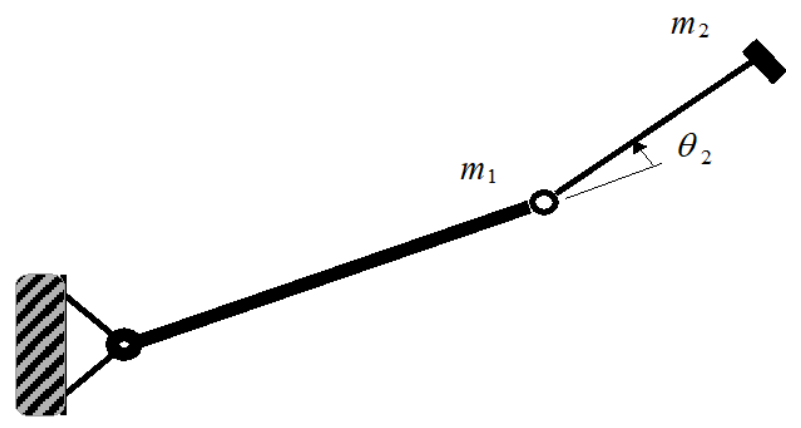

Fig. 1. Manipulator with rotational joints

The inertia matrix coefficients read as follows

$$
\begin{gathered}
\mathbf{M}_{11}=\left(m_{1}+m_{2}\right) l_{1}^{2}+m_{2} l_{2}^{2}+2 m_{2} l_{1} l_{2} c \\
\mathbf{M}_{12}=m_{2} l_{2}^{2}+m_{2} l_{1} l_{2} c, \mathbf{M}_{22}=m_{2} l_{2}^{2},
\end{gathered}
$$

where $l_{1}$ and $l_{2}$ are the links' lengths and $c=\cos \theta_{2}$. Then,

$$
\Delta=\left(l_{1}^{2} l_{2}^{2}\left(m_{1} m_{2}+m_{2}^{2} s^{2}\right)\right)^{-1}, s=\sin \boldsymbol{\theta}_{2}
$$

We can see that, with given mass of the first link $m_{1}$, the less the second link's mass $m_{2}$, the more capable for robust decentralized controllability of such manipulation systems are.

\subsection{Planar manipulator with three rotational joints}

The GDD-conditions, though not so restrictive, may not be satisfied in this case. Considering this linkage system in the worst (as regards the inertia interaction) case of stretched-out configuration, we have found that

(a) when the manipulator links are of equal length, the GDD condition for the inverse inertia matrix will be satisfied if the link masses strongly decrease in progressing from the base to the tip:

$$
m_{1} / m_{3}-16 m_{3} / m_{1}>16
$$

(b) if the link lengths decrease from the base to the tip, e.g., $l_{1}=2 l_{2}=4 l_{3}$ Fig. 2 , the decrease of link masses is not so strong as in case (a):

$$
4 m_{1} / m_{3}-8 m_{3} / m_{1}-9 m_{2} / m_{1}>14 \text {. }
$$

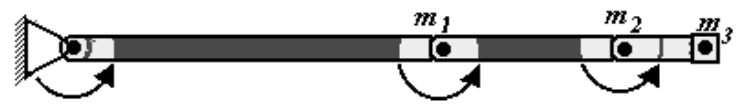

Fig. 2. Three-link manipulator 
Thus the stronger the decrease of link masses from the base to the tip, the better the capability of the manipulation systems (with either two or three joints) for independent joint controllability.

\subsection{Upright posture stabilisation}

This case-study is an extension of the study presented in [13], and now we consider a human body with assistive exoskeleton/exosuit. Unlike the usual adoption for ankle and hip control strategy, our proposal, from the controllability point of view, is that the main controlled joints are to be the ankle and the lumbar spine ones, Fig. 3a. By fixing (freezing) the hip joints and considering the pelvis and the legs as one (first) segment, the so-considered two-link manipulator system (hinged to the ground), Fig. 3b, [14], has much better controllability.

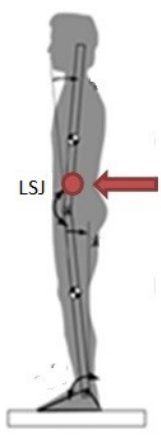

(a)

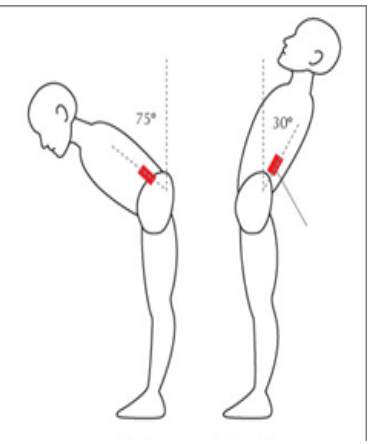

(b)

Fig. 3. Upright posture stabilization (LSJ is lumbar spine joint)

Thus, in upright postural control, human (with assistive device) has to take a strategy change from ankle to lumbar spine in response to increasing perturbation. Further on, our study can help designing robust balancing controller mimicking human control adaptation.

\section{Learning control and its optimisation}

The usual performance criteria for goal-directed movements are: the positioning accuracy, the movement execution time and the energy consumption. Once the control structure is defined, the next main stage in the control design is how to learn and optimize the specific control functions with a given point-to-point motion task. The main steps in our procedure for iterative motor control learning and optimization are as follows

1. Choose a set of appropriate test control functions: The term "appropriate" concerns the structure, shape, and bounds of the test control functions. Simple linear-spline control functions of "bang-pause-bang" or "bang-slope-bang" shape can be used for a first-order optimization. Parameters, describing the test control functions, like switch times, slopes, or pause lengths, mostly influence the reached position and the performance criteria.

2. Define the most relevant pairs of control parameters and control outputs: It means that for each controlled output we have to assign a control input, which mostly influences it. Regarding the controllability in multijoint tasks, the input-output pairs will be most relevant if couplings between these single-input single-output subsystems are to be as small as possible. 
3. Solve shooting equations and perform control parameter optimization: With the above input-output pairing, the given goal-directed motion task is transformed into systems of learning parameter equations with respect to the switch times. They are solved applying natural bisection algorithms and this is the first level of our control synthesis procedure. At the next level, the rest of the control parameters are varied to optimize the movement execution time and the energy consumption.

Following the above approach, we can find satisfactory suboptimal solutions with minimum number of control parameters to be learnt. Existence of feasible solutions and convergence of the bisection algorithms are guaranteed, which ensures feasibility and efficiency of our control learning procedure, [15]. This approach has been successfully applied to dynamics models of various-type CMS, e.g., planar manipulators, Fig. 4 and Fig. 5.

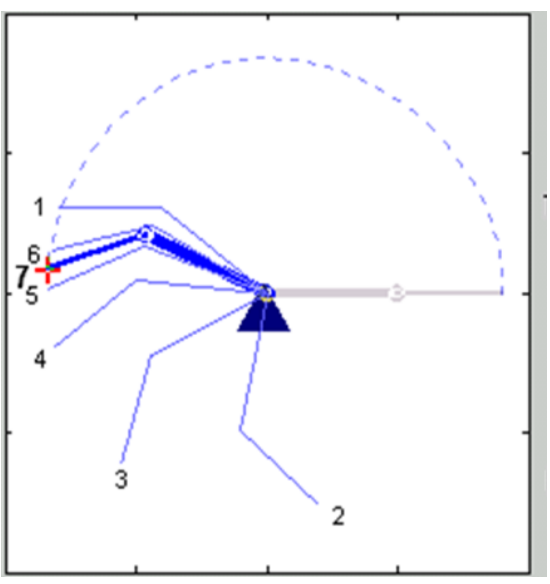

Fig. 4. Fast learning to reach a target

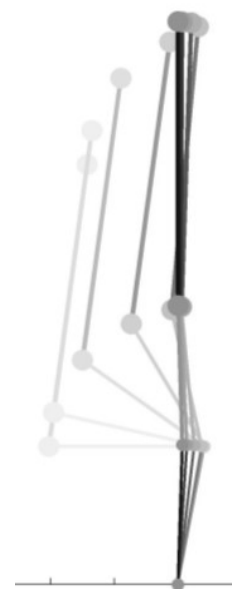

Fig. 5. Standing-up motion task

In case of wearable robotics, such movements can be generated by the corresponding muscles and/or by robotic orthoses (exoskeletons). The excitation signals naturally come from the brain and they may be processed via brain-computer interface $(\mathrm{BCI})$ and/or electro-myographical (EMG) devices.

\section{Conclusion}

We have proposed a generic conceptual framework for structure identification, analysis and design of control functions to achieve the best possible human/robot motion. It is based on underlying principles and advanced concepts from optimal control theory, neurophysiology, and controlled multi-segmental dynamics.

Recent studies at leading $R \& D$ centres for neurological rehabilitation say that three important principles must be observed: assistance-as-needed, embodied motor control and learning, and motivate patients for self-motor learning. Our control concepts and methodology for control design and optimal control learning are in accordance with these principles! The proposed concepts and algorithms could be very useful in designing and controlling arm/leg prostheses as well as in neuro-muscular rehabilitation of disabled people. Our current research intention is to extend the proposed approach to meet the next challenge: optimal control synthesis of 3D humanoid locomotion to produce efficient and naturally looking walking. 


\section{References}

1. J. Slotine, S. Shastry, Tracking control of nonlinear systems using sliding surfaces with application to robotic manipulators, I J Control, 2, 465-492 (1983)

2. P. Kiriazov, Robust Decentralized Control of Mechanical Systems, In: Solid Mechanics and its Applications, 52, (Ed. D. van Campen, Kluwer Acad. Publ., 175-182, 1997)

3. S. Riebe, H. Ulbrich, Model-based vibration isolation of a hexapod-system using a combined feedforward-feedback control concept, Proc. IEEE Conf. on Mechatronics and Robotics, 400-405 (2004)

4. R. Amirifar, N. Sadati, A low-order H-infinity controller design for an active suspension system via linear matrix inequalities, J. Vibr. and Cont, 10(8), 1181-1197 (2004)

5. L. H. Keel, S. P. Bhattacharyya, Robust, fragile, or optimal? IEEE Trans. on Autom. Cont., 42(8) 1098-1105 (1997)

6. W. Schiehlen, Multibody Systems Handbook (Berlin, Springer, 1990)

7. P. Kiriazov, Necessary and sufficient condition for robust decentralized controllability of robotic manipulators, American Control Conference, 2285-2287 (1994)

8. P. Kiriazov, Optimal robust controllers for multibody systems, Solid Mech. and its Applications, 30, (Ed. H. Ulbrich and W. Günthner, Springer-Verlag GmbH, 183-192, 2005)

9. S. Skogestad, I. Postlethwaithe, Multivariable Feedback Control: Analysis and Design, (Wiley, 1996)

10. P. Kiriazov, Efficient approach for dynamic parameter identification and control design of structronic systems, Solid Mechanics and its Applications, 89 (Eds. U. Gabbert and H. Tzou, Kluwer Publ, 323-330, 2001)

11. P. Kiriazov, Optimal robust controllers for multibody systems, Solid Mechanics and its Applications, 130, (Ed. by H. Ulbrich and W. Günthner, Springer-Verlag GmbH, 183192, 2005)

12. J. Lunze, Feedback Control of Large-Scale Systems (Prentice Hall, UK, 1992)

13. P. Kiriazov, Humanoid robots: how to achieve human-like motion, Humanoid 2003, 1$15,(2003)$

14. https://design.tutsplus.com/articles/human-anatomy-fundamentals-flexibility-and-joint$\underline{\text { limitations--vector-25401 }}$

15. K. Kiryazov , P. Kiriazov, Efficient learning approach for optimal control of human and robot motion, Emerging Trends in Mobile Robotics ( Eds: H. Fujimoto, M O Tokhi, H. Mochiyama, G. S. Virk, 1219-1227, 2010) 